\title{
Efficacy and tolerability of Amitriptyline versus Fluoxetine in the treatment of patients with somatoform disorder: A randomized comparative trial
}

\author{
Joshi S1, Pandey AK², Sapkota $\mathrm{N}^{3}$
}

1.Lecturer, Department of Psychiatry, PAHS, Lagankhel, Lalitpur, Nepal 2.Additional Professor, Department of Psychiatry, BPKIHS, Dharan, Nepal 3. Associate Professor, Department of Psychiatry, BPKIHS, Dharan, Nepal.

E-mail *Corresponding author: dr.sulochana@hotmail.com

\section{Abstract}

Introduction: Somatoform disorder(SFD) is a heterogeneous group of disorders with difficult diagnostic problems and skepticisms regarding the treatability. Though a common diagnosis among psychiatrists and physicians alike, there are limited studies on its treatment, more so in developing countries. Emerging evidences suggest that antidepressants may potentially be effective and reduce disability, costs, and patient dissatisfaction.

Objective: This study aims to compare the effectiveness of Amitriptyline and Fluoxetine in the treatment of patients with SFD presenting to department of Psychiatry at BPKIHS, Dharan.

Method: It is a hospital based open label, randomized comparative study which included 60 patients meeting ICD DCR criteria for SFD. The patients were assigned to Fluoxetine (30 patients) and Amitriptyline (30 patients) treatment groups as per block randomization and followed up for 8 weeks. The clinical effects were tested at baseline and subsequent evaluations with Patient Health Questionnaire-15(PHQ 15) and side effects enquired. Sociodemographic variables and other medical information were collected in a structured proforma.

Result: The reduction in PHQ 15 score was statistically significant in both the treatment groups. The reduction in PHQ 15 score was statistically faster in Fluoxetine group than Amitriptyline group from week 2 to week 4. Amitriptyline was more frequently associated with side effects than Fluoxetine.

Conclusion: Both Amitriptyline and Fluoxetine were comparable in effectiveness in SFD with Fluoxetine more tolerated than Amitriptyline.

Keywords: Amitryptilline, Fluoxetine, Somatoform

\section{INTRODUCTION}

Somatoform disorders(SFD) are characterized by preoccupation of physical complaints that occur in the absence of identifiable physical pathology ${ }^{1}$. It is a common health problem encompassing a variety of medical, social, psychological and economic burdens. The impact of the disease is experienced in all spheres of patient's life and indirectly in patient's family.

This disorder is inherent to many cultures worldwide and is often confused with factitious and malingering disorders ${ }^{1}$. It is one of the most frustrating disorders for clinicians due to diagnostic dilemma, high levels of patient dissatisfaction and management problems ${ }^{2,3}$. Though as common as depression and anxiety, the number and quality of trials studying the treatment of somatoform disorders are far less 4 . Most of the previous data are largely from TCAs and findings with SSRIs are relatively limited. However, recent data have shown Fluoxetine to be equally effective ${ }^{5}$. This study was undertaken to explore and compare the effectiveness of 
Amitriptyline with Fluoxetine in the patients with SFD.

\section{MATERIAL AND METHOD}

This study was a hospital based comparative prospective study conducted in patients with SFD. It was carried out in the department of Psychiatry at B.P. Koirala Institute of Health Sciences (BPKIHS), a tertiary-care teaching hospital at Dharan, Nepal for a period of 1year. Ethical clearance was obtained from the institutional review board. Informed consent was taken from the patients. Consecutive patients with SFD presenting at OPD of department of Psychiatry fulfilling the study criteria were included in the study. The study criteria included 18 to 60 years aged of either gender willing to participate in the study fulfilling the ICD DCR criteria for somatoform disorder without any comorbid disorder and/or other medication for somatic complaints.

Sixty patients who met the ICD DCR criteria for SFD were assigned to Fluoxetine (30 patients) and Amitriptyline (30 patients) treatment groups for 8 weeks by block randomization. Amitriptyline was started at $25 \mathrm{mg}$ per day at bed time and Fluoxetine at $20 \mathrm{mg}$ per day in the morning. Based on the clinical response obtained and tolerability, the treating team increased the dosage to maximum of $100 \mathrm{mg}$ per day of Amitriptyline and $40 \mathrm{mg}$ per day of Fluoxetine. No other medication was administered during the study period except for benzodiazepines, hypnotics, acetaminophen and proton pump inhibitors for temporary control of anxiety, insomnia and as needed for any other emergencies. The clinical effects were tested at baseline and subsequent evaluations with Patient Health Questionnaire-15 scale (PHQ 15) and side effects were enquired. All the cases underwent thorough clinical examination as per the study protocol.

Assessment for effectiveness and tolerability of medication was done at week: 2,4 and 8 . Sociodemographical profiles were noted using a selfdeveloped semi structured pro-forma. PHQ-15 is a self-administered, 15 items scale with scores $0-2$ where 0 (not bothered at all), 1 (bothered a little) and 2 (bothered a lot).The cutoff points for low, medium, and high somatic symptom severity is 5,10 and 15 respectively ${ }^{6}$. PHQ- 15 has been extensively used as an outcome measure of the severity of somatic symptoms and has been validated in over 6000 patients ${ }^{7,8,9}$. All the questionnaires were translated to Nepali by the principal investigator to help the patient report due to language difficulty.

Data analysis was done by Statistical Package for the Social Sciences (SPSS) version 17.0. Continuous variables were compared by student t-test. Other variables were described as frequency and proportion and were compared by Chi-Square test. Paired t-tests (before and after treatment administration) and independent samples t-tests were used for within group changes and comparison of treatment groups respectively.

\section{RESULT}

Total of 60 patients were studied in which $41.6 \%$ were from Sunsari district. The mean age was 35.17 years and 37.63 years for Amitriptyline and Fluoxetine group respectively with predominance of female $(63.3 \%)$. Majority were married $(83.3 \%)$, literate $(45 \%)$, Hindu $(86.7 \%)$ housewife $(41.7 \%)$ and from lower socioeconomic class (51. 7\%).Persistent Somatoform Pain Disorder $(30 \%)$ was the predominant variety of somatoform disorder in the study (Table 1). At baseline assessment, 32 out of 60 patients studied were in medium level and with treatment at the end of 8th week, 57 out of 60 patients studied had low level of the PHQ 15 score (Table 2).

The mean reductions in the PHQ 15 scores between the two treatment groups from baseline to subsequent evaluations at weeks 2,4 and 8 are shown in Table 3,4 and 5. The reduction of PHQ 15 scores were statistically significant and the reduction in score was faster for the Fluoxetine group compared to Amitriptyline group from week 2 to week 4 ( $p<0.041)$ (Table 6).

More than half of the patients (33 out of 60 ) developed one or more side effects with more patients in Amitriptyline treatment group developing side effects than the Fluoxetine treatment group (Figure 1) The most common side effects for Amitriptyline group was dry mouth and Fluoxetine group was GI disturbance. Others were dizziness, altered taste. 
Table No. 1: Clinicosocio-demographic profile of patients with somatoform disorder.

\begin{tabular}{|c|c|c|c|c|c|c|}
\hline \multirow[b]{3}{*}{ Characteristics } & \multirow[b]{3}{*}{ Category } & \multicolumn{2}{|c|}{ Subjects } & \multirow[b]{3}{*}{$\mathbf{n}$} & \multirow[b]{3}{*}{$\%$} & \multirow[b]{3}{*}{ P value } \\
\hline & & Amitriptyline & Fluoxetine & & & \\
\hline & & $n(\%)$ & n (\%) & & & \\
\hline \multirow[b]{3}{*}{ Age } & $<=30$ & $11(36.7)$ & $7(23.3)$ & 18 & 30 & \multirow[b]{3}{*}{0.363} \\
\hline & $31-40$ & $9(30)$ & $14(46.7)$ & 23 & 38.3 & \\
\hline & $>40$ & $10(33.3)$ & $9(30)$ & 19 & 31.7 & \\
\hline \multirow[b]{2}{*}{ Sex } & Male & $10(33.3)$ & $12(40)$ & 22 & 36.7 & \multirow[b]{2}{*}{0.592} \\
\hline & Female & $20(66.7)$ & $18(60)$ & 38 & 63.3 & \\
\hline \multirow[b]{2}{*}{ Marital Status } & Married & $26(86.7)$ & $24(80)$ & 50 & 83.3 & \multirow[b]{2}{*}{0.488} \\
\hline & Single & $4(13.3)$ & $6(20)$ & 10 & 16.7 & \\
\hline \multirow[b]{2}{*}{ Religion } & Hindu & $27(90)$ & $25(83.3)$ & 52 & 86.7 & \multirow[b]{2}{*}{0.448} \\
\hline & Others & $3(10)$ & $5(16.7)$ & 8 & 13.3 & \\
\hline \multirow[b]{3}{*}{ Education } & No Schooling & $8(26.7)$ & $10(33.3)$ & 18 & 30 & \multirow[b]{3}{*}{0.733} \\
\hline & Schooling & $15(50)$ & $12(40)$ & 27 & 45 & \\
\hline & Higher education & $7(23.3)$ & $8(26.7)$ & 15 & 25 & \\
\hline \multirow[b]{3}{*}{ Occupation } & Farmer & $5(16.7)$ & $9(30)$ & 14 & 23.3 & \multirow[b]{3}{*}{0.461} \\
\hline & Housewife & $14(46.7)$ & $11(36.7)$ & 25 & 41.7 & \\
\hline & Others & $11(36.7)$ & $10(33.3)$ & 21 & 35 & \\
\hline \multirow[b]{2}{*}{ Socioeconomic } & Lower & $16(53.3)$ & $15(50)$ & 31 & 51.7 & \multirow[b]{2}{*}{0.796} \\
\hline & Upper & $14(46.7)$ & $15(50)$ & 29 & 48.3 & \\
\hline \multirow[b]{8}{*}{ District } & Sunsari & $13(43.33)$ & $12(40)$ & 25 & 41.6 & \\
\hline & Morang & $5(16.66)$ & $2(6.66)$ & 7 & 11.7 & \\
\hline & Jhapa & $3(10)$ & $1(3.33)$ & 4 & 6.7 & \\
\hline & Saptari & $2(6.66)$ & $2(6.66)$ & 4 & 6.7 & \\
\hline & Siraha & $1(3.33)$ & $2(6.66)$ & 3 & 5 & \\
\hline & Khotang & $1(3.33)$ & $3(10)$ & 4 & 6.7 & \\
\hline & Bihar & $1(3.33)$ & $2(6.66)$ & 3 & 5 & \\
\hline & Others & $4(13.33)$ & $6(20)$ & 10 & 16.6 & \\
\hline \multirow[b]{7}{*}{ Diagnosis } & Somatization & $2(6.7)$ & $8(26.7)$ & 10 & 16.7 & \\
\hline & Undifferentiated & $2(6.7)$ & $3(10)$ & 5 & 8.3 & \\
\hline & Hypochondriasis & $2(6.7)$ & $5(16.7)$ & 7 & 11.7 & \\
\hline & Autonomic & $1(3.3)$ & $1(3.3)$ & 2 & 3.3 & \\
\hline & PSPD & $11(36.7)$ & $7(23.3)$ & 18 & 30 & \\
\hline & Others & $2(6.7)$ & $2(6.7)$ & 4 & 6.7 & \\
\hline & Unspecified & $10(33.3)$ & $4(13.3)$ & 14 & 23.3 & \\
\hline
\end{tabular}

Table no. 2: Distribution of PHQ 15 score severity in the Treatment Group

\begin{tabular}{|c|c|c|c|c|c|}
\hline \multirow{2}{*}{$\begin{array}{c}\text { PHQ } 15 \\
\text { Score }\end{array}$} & \multirow[b]{2}{*}{ Category } & \multicolumn{2}{|c|}{ Subjects } & \multirow[t]{2}{*}{ Frequency } & \multirow[t]{2}{*}{ Percent } \\
\hline & & Amitriptyline & Fluoxetine & & \\
\hline \multirow{3}{*}{$\begin{array}{c}\text { Baseline } \\
\text { score }\end{array}$} & Low & 6 & 5 & 11 & 18.3 \\
\hline & Medium & 17 & 15 & 32 & 53.3 \\
\hline & High & 7 & 10 & 17 & 28.3 \\
\hline \multirow{3}{*}{$\begin{array}{c}\text { Week } 2 \\
\text { score }\end{array}$} & Low & 25 & 18 & 43 & 71.7 \\
\hline & Medium & 5 & 9 & 14 & 23.3 \\
\hline & High & 0 & 3 & 3 & 5 \\
\hline \multirow{3}{*}{$\begin{array}{c}\text { Week } 4 \\
\text { score }\end{array}$} & Low & 29 & 25 & 54 & 90 \\
\hline & Medium & 1 & 4 & 5 & 8.3 \\
\hline & High & 0 & 1 & 1 & 1.7 \\
\hline \multirow{3}{*}{$\begin{array}{c}\text { Week } 8 \\
\text { score }\end{array}$} & Low & 29 & 28 & 57 & 95 \\
\hline & Medium & 1 & 2 & 3 & 5 \\
\hline & High & 0 & 0 & 0 & 0 \\
\hline
\end{tabular}


Table no. 3: Overall Mean of PHQ 15 score as per Treatment Group at each visit

\begin{tabular}{|c|c|c|c|}
\hline \multirow{2}{*}{ PHQ 15 Score } & \multicolumn{2}{|c|}{ Treatment Group } & \multirow{2}{*}{ P value } \\
\cline { 2 - 4 } & Amitriptyline (mean \pm SD) & Fluoxetine (mean \pm SD) & 0.047 \\
\hline PHQ 15 baseline & $7.87 \pm 2.968$ & $9.60 \pm 3.616$ & 0.023 \\
\hline PHQ 15week2 & $4.03 \pm 1.956$ & $5.57 \pm 2.991$ & 0.165 \\
\hline PHQ 15week4 & $2.77 \pm 1.794$ & $3.53 \pm 2.389$ & 0.517 \\
\hline PHQ 15week8 & $2.00 \pm 1.486$ & $2.27 \pm 1.680$ & \\
\hline
\end{tabular}

Table no. 4: Comparison of reduction of PHQ 15 score in Amitriptyline treatment group at each visit

\begin{tabular}{|c|c|c|c|c|c|c|c|}
\hline $\begin{array}{c}\text { Reduction of PHQ } \\
\text { 15 Score }\end{array}$ & Mean & \multirow{2}{*}{$\begin{array}{c}\text { Standard } \\
\text { Deviation }\end{array}$} & \multicolumn{2}{|c|}{$\begin{array}{c}\text { 95\% Confidence } \\
\text { Interval of the } \\
\text { Difference }\end{array}$} & \multirow{2}{*}{$\mathbf{t}$} & df & P value \\
\cline { 3 - 7 } & & & Lower & Upper & & & \\
\hline Baseline-week2 & 3.833 & 2.291 & 2.978 & 4.689 & 9.166 & 29 & .000 \\
\hline Week2 -week4 & 1.267 & 1.048 & .875 & 1.658 & 6.618 & 29 & .000 \\
\hline Week4-week8 & .767 & 1.165 & .332 & 1.202 & 3.604 & 29 & .001 \\
\hline
\end{tabular}

Table no. 5: Comparison of reduction of PHQ 15 score in Fluoxetine treatment group at each Visit

\begin{tabular}{|c|c|c|c|c|c|c|c|}
\hline $\begin{array}{c}\text { Reduction of PHQ } \\
\text { 15 Score }\end{array}$ & Mean & \multirow{2}{*}{$\begin{array}{c}\text { Standard } \\
\text { Deviation }\end{array}$} & \multicolumn{2}{|c|}{$\begin{array}{c}\text { 95\% Confidence } \\
\text { Interval of the } \\
\text { Difference }\end{array}$} & \multirow{2}{*}{$\mathbf{t}$} & df & P value \\
\cline { 3 - 7 } & & & Lower & Upper & & & \\
\hline Baseline-week2 & 4.033 & 2.498 & 3.101 & 4.966 & 8.844 & 29 & .000 \\
\hline Week2 -week4 & 2.033 & 1.712 & 1.394 & 2.672 & 6.506 & 29 & .000 \\
\hline Week4-week8 & 1.267 & 1.337 & .767 & 1.766 & 5.188 & 29 & .000 \\
\hline
\end{tabular}

Table no. 6: Comparison of reduction of PHQ 15 score between Amitriptyline and Fluoxetine treatment group

\begin{tabular}{|c|c|c|c|c|c|c|c|}
\hline \multirow[t]{2}{*}{$\begin{array}{l}\text { Reduction of } \\
\text { PHQ } 15 \text { score }\end{array}$} & \multirow[t]{2}{*}{$\mathbf{t}$} & \multirow[t]{2}{*}{ df } & \multirow[t]{2}{*}{ P value } & \multirow[t]{2}{*}{$\begin{array}{c}\text { Mean } \\
\text { Difference }\end{array}$} & \multirow{2}{*}{$\begin{array}{c}\text { Standard } \\
\text { Error } \\
\text { Difference }\end{array}$} & \multicolumn{2}{|c|}{$\begin{array}{c}\text { 95\% Confidence Interval of } \\
\text { the Difference }\end{array}$} \\
\hline & & & & & & Lower & Upper \\
\hline Baseline-week2 & -0.323 & 58 & 0.748 & -0.2000 & 0.61880 & -1.43866 & 1.03866 \\
\hline Week2 -week4 & -2.092 & 58 & 0.041 & -0.7667 & 0.36646 & -1.50021 & -0.03312 \\
\hline Week4-week8 & -1.544 & 58 & 0.128 & -0.5000 & 0.32383 & -1.14822 & 0.14822 \\
\hline
\end{tabular}

No serious adverse effects were reported in either group. None of the patient left the study due to side effects. Overall both treatments were well tolerated.

\section{DISCUSSION:}

Both Amitriptyline as well as Fluoxetine are effective for the treatment of SFD as shown by the statistically significant decrease in the total score of PHQ 15 from baseline to the endpoint. This is unlikely to be due to improvement in depressive or anxiety disorders as they were not included in the study. On head to head comparison between these two antidepressants, no agent was found to have statistically significant superior results than the other. The clinical benefits obtained is faster for the Fluoxetine group compared to Amitriptyline group from week 2 to week 4 . It remains unclear why differences were found between some treatment weeks but not others. However, similar trend in decline in PHQ 15 score was not consistently observed in subsequent evaluations. Although Amitriptyline is more frequently associated with side effects than Fluoxetine, no serious side effects were observed and both the agents were well tolerated. 
Available evidences from earlier studies support the use of both tricyclic antidepressants and SSRIs for the treatment of SFD. In a systematic review of 34 randomized clinical trials involving 3922 patients, 4 trials were found to have examined antidepressants in SFD. Beneficial effects of antidepressants in SFD was shown in 3 out of 4 trials $^{10}$. A meta-analysis of 94 randomized controlled trials of antidepressants found substantial benefit for treating SFD with improvement in patients occurring more than three times as compared with placebo (OR 3.4, 95\% CI 2.6-4.5).The meta-analysis also found that tricyclic antidepressants were beneficial in significantly more studies compared with SSRIs (76 versus 47 percent of studies), but there was an insufficient number of trials of SSRIs to draw a firm conclusion about the relative efficacy of the two classes of antidepressants ${ }^{11}$. In a study by Liu Hongxia ${ }^{12}$, clinical effects of both Fluoxetine and Amitriptyline were similar. However, Fluoxetine had less side effects than Amitriptyline. This finding is similar to our study in terms of side effect profile, however our study found Fluoxetine acting faster after $2^{\text {nd }}$ week till $4^{\text {th }}$ week.

In a study comparing Venlafaxine and Amitriptyline for SFD, no effective difference was observed in the two groups, but there was difference in the effective time of drugs at the end of 1 -week treatment ${ }^{13}$. Similar difference between the two treatment groups in effective time is shared by our study but differs in terms of duration, 1 week in this study and 2 weeks in ours. A double-blind randomized comparison study of Amitriptyline $25 \mathrm{mg}$ with placebo showed that Amitriptyline was effective in the treatment of chronic pain with significant differences within the first week ${ }^{14}$. This finding is contrary to our study as the effectiveness of Amitriptyline was observed early in the 1st week which could be due to the fixed low dose of Amitriptyline.

In a randomized, open-label trial of Fluoxetine versus sertraline in patients with undifferentiated somatoform disorder, both treatments were well tolerated with no serious adverse events ${ }^{15}$. This finding is in accordance with this study in terms of individual effectiveness by the treatment drug and the common side effect profile of Fluoxetine. A randomized double-blind placebo-controlled study of Fluoxetine in persistent somatoform pain disorder, a significant improvement was observed in Fluoxetine treatment group as compared to placebo group after 2 weeks of treatment ${ }^{16}$. The study effectiveness of Fluoxetine observed after 2 week of treatment is similar to our study.

As in earlier studies ${ }^{15,16,17}$ nausea or vomiting was the most common adverse effect of Fluoxetine in our study too but no patients left the study because of side effects as in those studies.

\section{LIMITATIONS:}

As the study was hospital based with small sample size, the findings could not be generalized. The PHQ 15 instrument used in the study is an English version, which caused difficulty in reporting due to language barrier and time consuming though a self-report questionnaire.

\section{CONCLUSION:}

This study shows that both Amitriptyline and Fluoxetine are significantly effective in treating SFDs with Fluoxetine apparently working faster than Amitriptyline from week 2 to week 4 of treatment initiation. Amitriptyline appears to be associated with side effects more frequently than Fluoxetine. This finding indicates that the somatoform patient can be treated with both of the medication safely. But for the optimal treatment further exploration into association between the effectiveness of treatment and factors affecting the treatment is needed.

\section{REFERENCES:}

1. Kaplan \& Saddock. Comprehensive Text book of Psychiatry. $8^{\text {thed. Philadelphia: }}$ Lippincott Williams and Wilkins; 2005. Volume 15, Chapter, Somatoform Disorders.

2. Kroenke K.Somatoform disorders and recent diagnostic controversies. Psychiatric Clinic of North America. 2007 Dec; 30(4):593-619.

3. R. K. Chadda, M. S. Bhatia, S. Shome and K. N. Thakur.Psychosocial Dysfunction in Somatizing Patients. 
British Journal of Psychiatry. 1993;163, 510-513.

4. Muller JE, Wentzel I, Koen L, Niehaus DJ, Seedat S, Stein DJ. Escitalopram in the treatment of multisomatoform disorder: a double-blind, placebocontrolled trial. Int Clin Psychopharmacol. 2008; 23(1):43-48.

5. Winfried Rief, PH.D.,WolfgangHiller et al.A Two-Year Follow-Up Study of Patients with Somatoform Disorders. The Academy of Psychosomatic Medicine 1995; 36:376-386

6. Lipowski ZJ. Somatization: the experience and communication of psychological distress as somatic symptoms. Psychother Psychosom. 1987;47(3-4):160-7.

7. Kroenke K, Spitzer RL, Williams JBW. The PHQ-15: validity of a new measure for evaluating the severity of somatic symptoms. Psychosom Med 2002;64:258-66.

8. Kroenke K. Physical symptom disorder: a simpler diagnostic category for somatization-spectrum conditions. J Psychosom Res 2006;60:335-9.

9. Stephen M Stahl: Essential Psychopharmacology, 3rd Edition. Cambridge University Press, 2000. Chapter 12 , Antidepressant; 511-600.

10. Kroenka K. Efficacy of treatment of somatoform disorders: A review of randomized controlled trials. Psychosomatic Medicine. 2007; 69:881888.

11. O'Malley PG, Jackson JL, Santoro J, et al. Antidepressant therapy for unexplained symptoms and symptom syndromes. J Fam Pract.1999; 48: 980-990.

12. Liu Hongxia. A comparative study of fluoxetine and amitriptyline in the treatment of somatoform disorders. Shandong Archives of Psychiatry.200304 .

13. Jiang Xiao-qin, Yang Kai-ren, HU Weiming, et al. A Clinical Effective Analysis of Venlafaxine in Treatment of Somatoform Disorders; Chinese general practice.2005-14.

14. McQuay HJ, Carroll D, Glynn CJ. Low dose amitriptyline in the treatment of chronic pain. Anaesthesia .1992;47:64652.

15. Changsu Han, Chi-Un Paeet al: Fluoxetine versus sertraline in the treatment of patients with undifferentiated somatoform disorder: A randomized, open-label, 12-week, parallel-group trial. Progress in NeuroPsychopharmacology \& Biological Psychiatry. 2008; 32 :437-444.

16. Yan-Li Luo, Ming-Yuan Zhang et al: A randomized double-blind clinical trial on analgesic efficacy of fluoxetine for persistent somatoform pain disorder. Progress in Neuro-Psychopharmacology \& Biological. 2009; 33:1522-1525

17. Wernicke, J. F. (1985) The side effect profile and safety of fluoxetine. Journal of Clinical Psychiatry. 1985;46:59-67. 